\title{
cDNA cloning confirms the polyadenylation of RNA decay intermediates in Streptomyces coelicolor
}

\author{
Patricia Bralley and George H. Jones
}

Department of Biology, Emory University, Atlanta, GA 30322, USA
Author for correspondence: Patricia Bralley. Tel: +1 404727 4208. Fax: +1 4047272880. e-mail: pbralley@biology.emory.edu

In Escherichia coli the poly(A) tails of messenger and rRNAs are a major determinant of RNA stability. These tails are formed primarily by poly(A) polymerase I (PAP I) in wild-type strains or by polynucleotide phosphorylase (PNPase) in PAP I-deficient strains. In Streptomyces coelicolor it has been shown that mycelial RNAs display biochemical characteristics consistent with the presence of poly(A) tails. To confirm the occurrence of polyadenylation, rRNA and mRNA transcripts from S. coelicolor were isolated by oligo(dT)dependent RT-PCR followed by CDNA cloning. One of the clones obtained was polyadenylated at a site corresponding to the mature $3^{\prime}$ terminus of 16S rRNA, while two 235 rRNA cDNA clones were polyadenylated at precursor processing sites. Other clones identified polyadenylation sites internal to the coding regions of both 165 and 235 rRNAs, and redD and actII-orf4 mRNAs. While most rRNA CDNA clones displayed adenosine homopolymer tails, the poly(A) tails of three rRNAs and all the redD and actII-orf4 clones consisted of a variety of heteropolymers. These results suggest that the enzyme primarily responsible for polyadenylation in S. coelicolor is PNPase rather than a PAP I homologue.

Keywords: redD, actII-orf4, polynucleotide phosphorylase, poly(A) polymerase

\section{INTRODUCTION}

In Escherichia coli, polyadenylation of RNA is a key determinant of RNA stability. Degradation is initiated by endonucleases, primarily RNase E, and to a lesser extent by RNase G and RNase III (reviewed by Steege, 2000). RNase E cleaves at specific AU-rich, singlestranded sites in both mRNA and rRNA (Bessarab et al., 1998). Endonucleolytic cleavage is followed by polyadenylation and digestion of poly(A) tails by exonucleases such as RNase II and polynucleotide phosphorylase (PNPase) (Steege, 2000). Poly(A) polymerase I (PAP I) is the enzyme primarily responsible for addition of poly(A) tails (Cao \& Sarkar, 1992). Surprisingly, PNPase has been shown to function also as a poly $(\mathrm{A})$ polymerase in E. coli, synthesizing poly(A) tails that are heteropolymers and accounting for the residual polyadenylation activity seen in PAP I-deficient strains (Mohanty \& Kushner, 2000).

Relatively little is known regarding RNA processing in the Gram-positive streptomycetes which produce over

Abbreviations: PAP I, poly(A) polymerase I; PNPase, polynucleotide phosphorylase.
$70 \%$ of the world's antibiotics (Bibb, 1996). However, homologues of several E. coli endo- and exonucleases have been identified. An RNase E homologue has been found in Streptomyces coelicolor and Streptomyces lividans (Hagege \& Cohen, 1997). The absB locus of $S$. coelicolor appears to encode an RNase III homologue. Mutation of $a b s B$ abolishes antibiotic production (Price et al., 1999). In Streptomyces antibioticus the pnp gene encodes an enzyme that functions as both a PNPase and a guanosine pentaphosphate synthetase (Jones \& Bibb, 1996). Guanosine pentaphosphate, pppGpp, is the precursor of ppGpp, a compound that plays a critical role in initiation of antibiotic synthesis in streptomycetes (Chakraburrty \& Bibb, 1997; Hoyt \& Jones, 1999).

To establish a foundation for studies of the relationship between RNA metabolism and antibiotic production, a poly(A) polymerase activity was recently demonstrated in crude mycelial extracts of S. coelicolor (Bralley \& Jones, 2001). Digestion of S. coelicolor total RNA with RNase A and T1 revealed a maximum poly(A) tail length of $18 \mathrm{nt}$ when the tails were resolved by PAGE. Amplification of RNA species, retained on oligo(dT) cellulose, by RT-PCR, produced cDNA corresponding to $p n p$, the sigma factor, $h r d B$, and the pathway-specific 
regulators of antibiotic synthesis, redD and actII-orf4. Here we verify the presence of poly (A) tails in total RNA samples by cloning and sequencing cDNA clones of specific mRNA and rRNA genes. The presence of heteropolymeric tails in these clones suggests that in $S$. coelicolor the major poly (A) polymerase is PNPase.

\section{METHODS}

Growth of organisms and isolation of RNA. S. coelicolor M145 was grown at $30^{\circ} \mathrm{C}$ for $24 \mathrm{~h}$ in SMM medium as described by Kieser et al. (2000). Total mycelial RNA was isolated as described by Hsieh \& Jones (1995). The quantity and quality of RNA were checked by spectrophotometry and agarose gel electrophoresis. To ensure the absence of contaminating DNA in the RT-PCR, it was necessary to treat the total RNA twice with RQ1 RNase-free DNase, following the protocol of the manufacturer (Promega).

RT-PCR. Five micrograms of total RNA was reversetranscribed using 2 pmol primer ADoT (5'-GGATCCGAATTCTCTAGAGCT $17{ }^{-} 3^{\prime}$ ) and 200 units Superscript II (Gibco$\mathrm{BRL})$, following the extension protocol of the manufacturer. The $100 \mu \mathrm{l}$ PCR reaction consisted of $5 \mu \mathrm{l}$ R reaction mix, 2 units Vent (exo-) DNA polymerase (New England Biolabs), $10 \mu \mathrm{l}$ DMSO, $5 \mu \mathrm{l} 100 \mathrm{mM} \mathrm{MgSO}, 5 \mu \mathrm{l} 5 \mathrm{mM}$ dNTPs, $10 \mu \mathrm{l}$ $10 \times$ buffer, as well as $20 \mathrm{pmol}$ of both a gene-specific primer and an adapter primer, AD20 (5'-GGATCCGAATTCTCTAGAGC-3'), consisting of sequences contained in ADoT. Typically, gene-specific primers were designed as sequences internal to the gene of interest and some 300-600 bases upstream of the $3^{\prime}$ end of the gene. The gene-specific primers were: red440, 5'-CCGTCGAACTGCTGGAGACC-3', for redD; act520, 5'-GACGAGCGACGACCGGGAATTG-3', for actII-orf4; 16S1, 5'-CTGTCGTCGCTCGTGTCGTG-3', for $16 \mathrm{~S}$ rRNA; and 23S1750, 5'-GATGCCGAGCCGGATTGTGGTG-3', as well as 23S2700, 5'-CATATCGACGGGATGGTTTGG-3', for 23S rRNA. This last primer was designed to hybridize significantly closer to the $3^{\prime}$ end of the gene than 23S1750 and was used to isolate clones 3134 and 3128. The PCR reaction consisted of an incubation at $94^{\circ} \mathrm{C}$ for $1 \mathrm{~min}$, followed by 30 cycles consisting of $30 \mathrm{~s}$ at $90^{\circ} \mathrm{C}, 1 \mathrm{~min}$ at $50{ }^{\circ} \mathrm{C}$ and $1 \mathrm{~min}$ at $72^{\circ} \mathrm{C}$, and ended with extension at $72{ }^{\circ} \mathrm{C}$ for $10 \mathrm{~min}$.

Cloning and sequencing of cDNA. Four microlitres of RTPCR product was added to the Topo-TA Cloning System, following the protocol of the manufacturer (Invitrogen). $\beta$ Galactosidase $\alpha$-complementation was used for selection and isolation of clones, which were subsequently analysed by restriction enzyme digestion. Candidate clones were then sequenced by the Emory University DNA Sequencing Core Facility.

\section{RESULTS AND DISCUSSION}

\section{Identification of heteropolymeric and homopolymeric poly(A) tails in S. coelicolor rRNA and mRNA CDNA clones}

Previously, it was shown that streptomycete RNA displays many of the biochemical characteristics expected of polyadenylated RNA (Bralley \& Jones, 2001). Here, we confirm the presence of polyadenylated tails in S. coelicolor through oligo(dT)-dependent RTPCR. Utilizing $5^{\prime}$ gene-specific primers and a common 3'
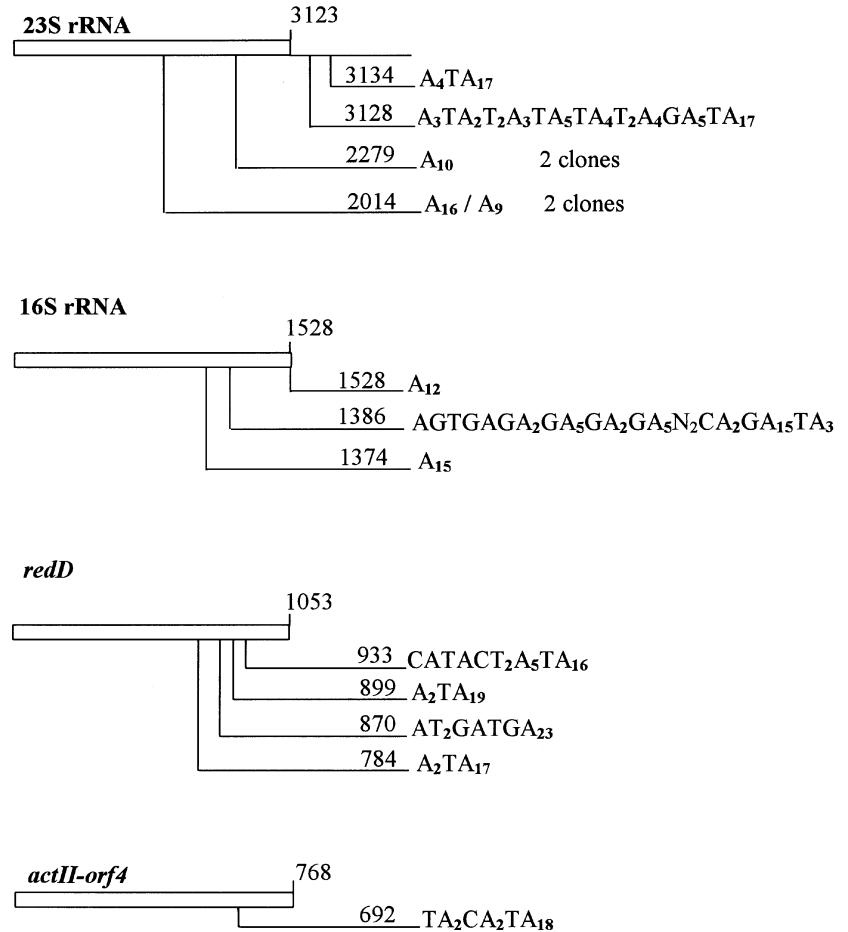

Fig. 1. Sites and composition of mRNA and rRNA poly(A) tails sequenced from CDNA clones isolated by RT-PCR of total RNA from $S$. coelicolor. The position of polyadenylation of each tail (not drawn to scale) is reckoned from the translation start codon of mRNA ORFs or from the mature 5 ' end of rRNA genes and is used to designate the number of each clone. The number of the last nucleotide of the mature rRNA or translation stop codon of mRNA is indicated at the top right corner of each box.

adapter primer, cDNA clones were constructed from the transcripts of two genes that regulate antibiotic production, redD and actII-orf4, and from $16 \mathrm{~S}$ and $23 \mathrm{~S}$ rRNAs. While this method does not allow determination of the maximum length of poly(A) tails, it was nevertheless possible to isolate cDNAs corresponding to polyadenylated RNA species. Sequencing of the cDNA clones revealed an unexpected pattern of polyadenylation. All the redD and actII-orf4 cDNA clones and three rRNA cDNA clones possessed highly heteropolymeric tails, composed of approximately $20 \% \mathrm{G}$, T and $\mathrm{C}$ residues (Fig. 1).

This heterogeneity is confined to the $5^{\prime}$ end of the tails, while the $3^{\prime}$ ends consist of poly(A) (Fig. 1). It is possible that this pattern of hetero- and homopolymeric sequences is caused by the addition of nucleotides by more than one enzyme. However, we feel it is more likely that the $3^{\prime}$ sequences result from the hybridization of the oligo $(\mathrm{dT})_{17}$ primer, used for reverse transcription, to A-rich yet heteropolymeric sequences, thus converting the $3^{\prime}$ sequences to homopolymers prior to cDNA cloning. Mohanty \& Kushner (2000) reported a similar phenomenon in experiments performed in E. coli.

Fig. 1 also reveals an apparent difference in tail composition of the mRNAs and rRNAs. The mRNA 
cDNA clones are all heteropolymers, while the majority of the rRNA cDNA clones are relatively short, adenosine homopolymers. However, if the oligo $(\mathrm{dT})_{17}$ primer is indeed hybridizing to A-rich heteropolymeric tails and converting the sequences during reverse transcription, short homopolymeric tails are still possible and even to be expected. The fact that the two longest heteropolymeric tails were detected in the rRNA clones supports this hypothesis.

Our results are consistent with the conclusion that, unlike the situation in E. coli, heteropolymeric poly(A) tails are the rule, rather than the exception in S. coelicolor. Furthermore, if the adenosine homopolymeric stretches at the $3^{\prime}$ end are created by the oligo(dT) primer during reverse transcription, then we can revise the GCT content of the tails to about $40 \%$. A high GCT content has been reported in tails polymerized by polynucleotide phosphorylase (see below).

\section{Analysis of polyadenylation sites}

One $16 \mathrm{~S}$ rRNA clone (1528) was polyadenylated at a site corresponding to the $3^{\prime}$ end of the mature rRNA. Two $23 \mathrm{~S}$ rRNA clones (3134 and 3128) were polyadenylated at $3^{\prime}$ immature precursor sites similar to those found in E. coli (Mohanty \& Kushner, 1999, 2000). All the other clones showed polyadenylation deep within the coding regions (Fig. 1). Similar internal locations of polyadenylation sites have been reported in the bacteriophage T7 (Johnson et al., 1998) and in the E. coli rpsO gene (Haugel-Nielsen et al., 1996). This last result suggests that polyadenylation occurred after either extensive degradation by exonucleases or after endonucleolytic cleavage.

Degradation of mRNA in E. coli is initiated by one or more cleavages by endonucleases followed by $3^{\prime} \rightarrow 5^{\prime}$ digestion by exonucleases (PNPase and RNase II). In E. coli the predominant mRNA endonuclease is RNase E (Steege, 2000). Since RNase E appears to cut at sites that are AU-rich and single-stranded (Coburn \& Mackie, 1999), and since Bessarab et al. (1998) have also shown that the degradosome contains fragments of rRNA that are cut by RNase E, we examined our cDNA clones for such sequences.

The AU content of the 10 bases covering each putative cleavage site is shown in Table 1 . The cleavage sites of the rRNAs typically displayed a higher AU content than the mRNAs. This higher AU content of the rRNAs is explained in part by the lower GC content of these genes (mean $56 \mathrm{~mol} \%$ ) compared to that of redD and actIIorf4 (mean $68 \mathrm{~mol} \%$ ). Analysis of the secondary structures of the two transcripts with the highest AU content (16S 1528 and 16S 1374) also showed that the putative cleavage sites lie in single-stranded regions (Fig. 2). These two transcripts thus appear to possess RNase E-like processing sites that were immediately polyadenylated following cleavage. However, additional biochemical analysis will be necessary to confirm the identity of the enzyme responsible for cleavage. Note
Table 1. The AU content of the sequence surrounding the polyadenylation sites of rRNA and mRNAs

\begin{tabular}{|c|c|c|}
\hline RNA & AU content & Polyadenylation site \\
\hline \multicolumn{3}{|l|}{ rRNA } \\
\hline 16S 1528 & $7 / 10$ & UUUCU · AAGGA \\
\hline 16S 1374 & $6 / 10$ & CUUGU $\cdot$ ACACA \\
\hline 2352279 & $5 / 10$ & AGGAC $\cdot$ GGAAA $†$ \\
\hline $23 S 3128$ & $4 / 10$ & AGUUG $\cdot$ CUCGC \\
\hline $23 S 3134$ & $3 / 10$ & UCGCG $\cdot$ UCCAC \\
\hline $23 S 2014$ & $3 / 10$ & CGUGC.GAAGC + \\
\hline 16S 1386 & $2 / 10$ & GCCCG $\cdot$ UCACG \\
\hline \multicolumn{3}{|l|}{ mRNA } \\
\hline actII-orf4 692 & $4 / 10$ & CGACG $\cdot$ AACUG \\
\hline redD 784 & $4 / 10$ & CAUGC $\cdot$ UCCAG \\
\hline redD 899 & $3 / 10$ & GCCAG·GAAGC \\
\hline redD 870 & $3 / 10$ & AGGGA·GACGC \\
\hline redD 933 & $1 / 10$ & AGCCG ·GGCGC \\
\hline
\end{tabular}

* The sequence is read $5^{\prime} \rightarrow 3^{\prime}$ with the poly(A) attachment site indicated by '.'. Nucleotides downstream of - are thus missing from the cDNA clones.

†Two cDNA clones with the same polyadenylation sites were isolated in independent RT-PCR experiments.

that the third 16S rRNA cleavage site (clone 1386), which displays the lowest AU content, occurs in a double-stranded region. A homologue of RNase III, an enzyme that cuts dsRNA, has been identified at the abs locus in S. coelicolor (Price et al., 1999). The putative cleavage sites in the $23 \mathrm{~S}$ rRNA and mRNA transcripts we have identified thus far do not appear similar to sequences recognized by RNase E.

\section{Sequence analysis of poly(A) tails suggests PNPase is the primary polyadenylation enzyme in streptomycetes}

Applying similar RT-PCR methods to those used here, Mohanty \& Kushner (1999) showed in E. coli that 6/8 lpp clones were homopolymers, while two clones contained one $\mathrm{T}$ and $\mathrm{C}$ residue, respectively. A similar predominance of high A content tails has been reported for the flagellin mRNA of Bacillus subtilis and bacteriophage T7 mRNA (Cao \& Sarkar, 1993, Johnson et al., 1998). In E. coli, the extent to which tails are heteropolymers is directly related to the activity of PNPase. In strains deficient in PAP I, lpp poly(A) tails were heteropolymers and remarkably like those seen here in wild-type $S$. coelicolor. There was also a shift from homo- to heteropolymers when PNP was overexpressed in a PAP I-deficient strain. By contrast, when PAP I was overexpressed in a PNPase-deficient strain, tails consisted of homopolymer sequences (Mohanty \& Kushner, 2000). Ipp displayed only homopolymer tails when clones were isolated from strains deficient in the exoribonucleases PNPase and RNase II (Cao \& Sarkar, 1992). 
(a)

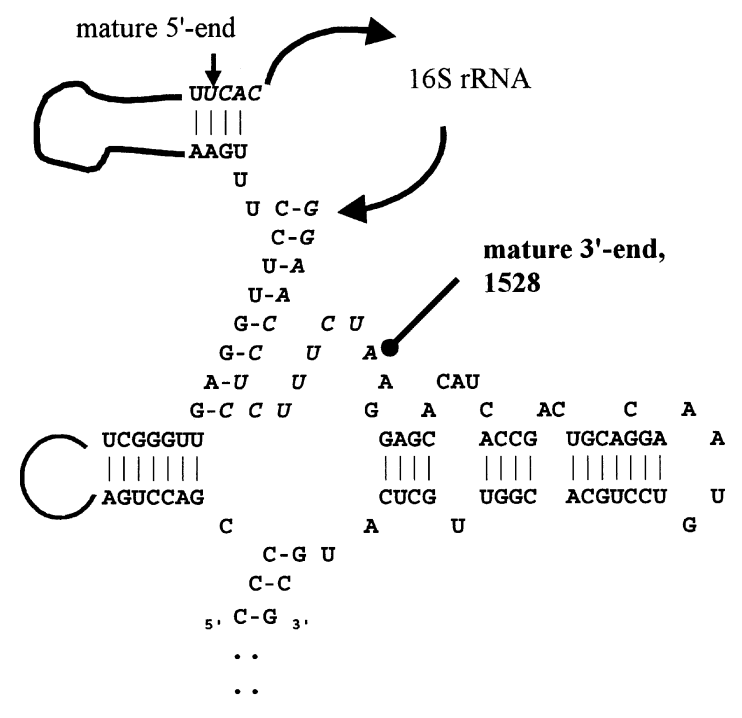

(b)

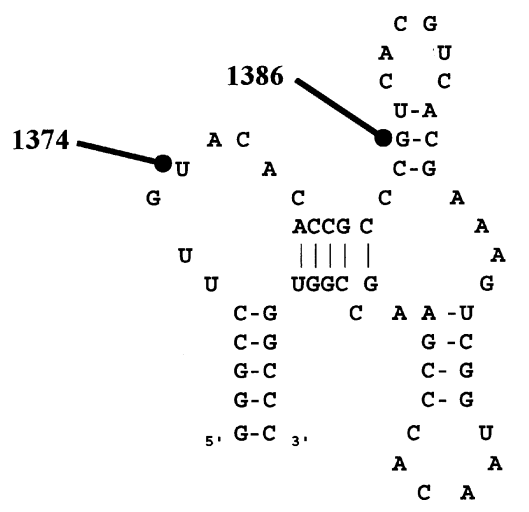

Fig. 2. Potential secondary structures of the RNA corresponding to $16 \mathrm{~S}$ rRNA CDNA clones. (a) The structure of the putative cleavage site in 1528 adapted from Baylis \& Bibb's (1988) folding of sequences flanking the unprocessed 16S rRNA. Mature 16S rRNA sequences are shown in italics. The numbering system is based upon the mature 16S rRNA sequence contained in the $r r n B$ operon of the $S$. coelicolor cosmid 151 (GenBank accession no. AL109848). (b) The secondary structures of sequences containing the putative cleavage sites in clones 1374 and 1386 as predicted by the Lasergene GeneQuest program 4.05 (DNASTAR). All of the bases shown are within the mature rRNA.

In spinach chloroplasts, heterologous poly(A) tails similar to those of $S$. coelicolor have been isolated by Lisitsky et al. (1996). cDNA clones of the psbA gene displayed heteropolymeric tails that consisted of $70 \%$ $\mathrm{A}, 25 \% \mathrm{G}$ and $5 \% \mathrm{C}$ and $\mathrm{T}$. Interestingly, attempts to purify a PAP I homologue from spinach chloroplasts, by following in vitro polyadenylation activity, resulted in the isolation of only PNPase. It is now thought that no E. coli PAP I homologue exists in spinach chloroplasts and that both polyadenylation and exonucleolytic degradation are carried out by PNPase (Yehudai-Resheff et al., 2001).
A similar picture appears to be emerging in S. coelicolor. To date, attempts to identify a PAP I homologue in streptomycetes have failed. Theoretically, three enzymes, PNPase, tRNA nucleotidyltransferase and RNase PH, could function as a PAP (Mohanty \& Kushner, 2000). A computer search of the $S$. coelicolor genome for a PAP I homologue identified only one ORF, H24.18. This sequence shows $48 \%$ similarity to E. coli PAP I (Bralley \& Jones, 2001). However, ORF H24.18 has even greater similarity to a protein from $B$. subtilis $(53 \%)$ that has already been shown to be a tRNA nucleotidyltransferase (Raynal et al., 1998). We speculate that ORF H24.18 also encodes a tRNA nucleotidyltransferase. A homologue of RNase PH (SCE19A.04c) with $61 \%$ identity to the E. coli protein was identified by the $S$. coelicolor genome sequencing project (www.sanger.ac.uk/Projects/S_coelicolor). However, overexpression of $\mathrm{RNase} \mathrm{PH}$ in E. coli resulted in shorter poly (A) tails, suggesting that it does not serve as a polymerase in that organism (Mohanty \& Kushner, 2000). Since PNPase is known to be a poly(A) polymerase in both E. coli and chloroplasts (Mohanty \& Kushner, 2000; Yehudai-Resheff et al., 2001), we feel it is currently the most likely candidate for that role in streptomycetes. The fact that numerous and varied attempts to create a $p n p$ knockout in two streptomycete strains have failed (G. H. Jones, unpublished results) suggests that the gene is essential in streptomycetes because it does serve as both the major poly(A) polymerase and an exonuclease. We are currently exploring this possibility.

\section{ACKNOWLEDGEMENTS}

This work was supported by a grant from the Emory University Research Committee.

\section{REFERENCES}

Bayliss, H. A. \& Bibb, M. J. (1988). Transcriptional analysis of the $16 \mathrm{~S}$ rRNA gene of the $r r n D$ gene set of Streptomyces coelicolor A3(2). Mol Microbiol 2, 569-579.

Bessarab, D. A., Kaberdin, V. R., Wei, C.-L., Liou, G.-G. \& Lin-Chao, S. (1998). RNA components of Escherichia coli degradosome: Evidence for rRNA decay. Proc Natl Acad Sci USA 95, 3157-3161.

Bibb, M. (1996). The regulation of antibiotic production in Streptomyces coelicolor A3(2). Microbiology 142, 1335-1344.

Bralley, P. \& Jones, G. H. (2001). Poly(A) polymerase activity and RNA polyadenylation in Streptomyces coelicolor A3(2). Mol Microbiol 40, 1155-1164.

Cao, G.-J. \& Sarkar, N. (1992). Poly(A) RNA in Escherichia coli: nucleotide sequence at the junction of the $l p p$ transcript and the polyadenylate moiety. Proc Natl Acad Sci US A 89, 7546-7550.

Cao, G.-J. \& Sarkar, N. (1993). Poly(A) RNA in Bacillus subtilis: identification of the polyadenylation site of flagellin mRNA. FEMS Microbiol Lett 108, 281-286.

Chakraburrty, R. \& Bibb, M. J. (1997). The ppGpp synthetase gene (relA) of Streptomyces coelicolor A(3)2 plays a conditional role in antibiotic production and morphological differentiation. J Bacteriol 179, 5854-5861. 
Coburn, G. A. \& Mackie, G. A. (1999). Degradation of mRNA in Escherichia coli: an old problem with some new twists. Prog Nucleic Acid Res Mol Biol 62, 55-108.

Hagege, J. M. \& Cohen, S. N. (1997). A developmentally regulated Streptomyces endoribonuclease resembles ribonuclease E of Escherichia coli. Mol Microbiol 25, 1077-1090.

Haugel-Nielsen, J., Hajnsdorf, E. \& Regnier, P. (1996). The $r p s O$ mRNA of Escherichia coli is polyadenylated at multiple sites resulting from endonucleolytic processing and exonucleolytic degradation. EMBO J 15, 3144-3152.

Hoyt, S. \& Jones, G. H. (1999). relA is required for antibiotic production in Streptomyces antibioticus. J Bacteriol 181, 3824-3829.

Hsieh, C. J. \& Jones, G. H. (1995). Nucleotide sequence, transcriptional analysis and glucose regulation of the phenoxazinone synthetase gene (phsA) from Streptomyces antibioticus. J Bacteriol 177, 5740-5747.

Johnson, M. D., Popowski, J., Cao, G.-J., Shen, P. \& Sarkar, N. (1998). Bacteriophage T7 mRNA is polyadenylated. Mol Microbiol 27, 23-30.

Jones, G. H. \& Bibb, M. J. (1996). Guanosine pentaphosphate synthetase from Streptomyces antibioticus is also a polynucleotide phosphorylase. J Bacteriol 176, 1482-1487.

Kieser, T., Bibb, M. J., Buttner, M. J., Chater, C. F. \& Hopwood, D. A. (2000). Practical Streptomyces Genetics. Norwich: John Innes Foundation.
Lisitsky, I., Klaff, P. \& Schuster, G. (1996). Addition of destabilizing poly $(\mathrm{A})$-rich sequences to endonuclease cleavage sites during the degradation of chloroplast mRNA. Proc Natl Acad Sci U S A 93, 13393-13403.

Mohanty, B. K. \& Kushner, S. R. (1999). Analysis of the function of Escherichia coli poly(A) polymerase I in RNA metabolism. Mol Microbiol 34, 1094-1108.

Mohanty, B. K. \& Kushner, S. R. (2000). Polynucleotide phosphorylase functions both as a $3^{\prime} \rightarrow 5^{\prime}$ exonuclease and a poly(A) polymerase in Escherichia coli. Proc Natl Acad Sci USA 97, 11966-11971.

Price, B., Adamidis, T., Kong, R. \& Champness, W. (1999). A Streptomyces coelicolor antibiotic regulatory gene, absB, encodes an RNase III homolog. J Bacteriol 181, 6142-6151.

Raynal, L. C., Kirsch, H. M. \& Carpousis, A. J. (1998). The Bacillus subtilis nucleotidyltransferase is a tRNA CCA-adding enzyme. J Bacteriol 180, 6276-6282.

Steege, D. A. (2000). Emerging features of mRNA decay in bacteria. RNA 6, 1079-1090.

Yehudai-Resheff, S., Hirsh, M. \& Schuster, G. (2001). Polynucleotide phosphorylase functions as both an exonuclease and a poly(A) polymerase in spinach chloroplasts. Mol Cell Biol 21, 5408-5416.

Received 12 October 2001; revised 11 December 2001; accepted 3 January 2002. 